\title{
AUV Benthic Habitat Mapping in South Eastern Tasmania
}

\author{
Stefan B. Williams, Oscar Pizarro, Michael Jakuba, Neville Barrett, Paper-ID 48
}

\begin{abstract}
This paper describes a two week deployment of the Autonomous Underwater Vehicle (AUV) Sirius on the Tasman Peninsula in SE Tasmania and in the Huon Marine Protected Area (MPA) to the South West of Hobart. The objective of the deployments described in this work were to document biological assemblages associated with rocky reef systems in shelf waters beyond normal diving depths. At each location, multiple reefs were surveyed at a range of depths from approximately $50 \mathrm{~m}$ to $100 \mathrm{~m}$ depth. We illustrate how the AUV based imaging complements benthic habitat assessments to be made based on the ship-borne swath bathymetry. Over the course of the 10 days of operation, 19 dives were undertaken with the AUV covering in excess of 70 linear kilometers of survey and returning nearly 160,000 geo-referenced high resolution stereo image pairs. These are now being analysed to describe the distribution of benthic habitats in more detail.
\end{abstract}

\section{Introduction}

The Autonomous Underwater Vehicle (AUV) Sirius was part of a two week expedition in October, 2008, whose objective was to describe biological assemblages associated with rock reef systems in deep shelf waters on the Tasman Peninsula in SE Tasmania and in the Huon Marine Protected Area (MPA) to the South West of Hobart. Detailed multibeam sonar bathymetry data were previously collected by Geoscience Australia using a Simard EM3002 multibeam sonar system, Applannix motion sensor and C-Nav GPS to provide high-resolution Digital Elevation Maps

Stefan B. Williams, Oscar Pizarro and Michael Jakuba

Australian Centre for Field Robotics, Uni. of Sydney, Sydney, NSW 2006, Australia

e-mail: stefanw, o.pizarro, m.jakuba@acfr.usyd.edu.au

Neville Barrett

Tasmanian Aquaculture and Fisheries Institute, Uni. of Tasmania, Hobart, Tas 7001, Australia

e-mail: Neville.Barrett@utas.edu.au 


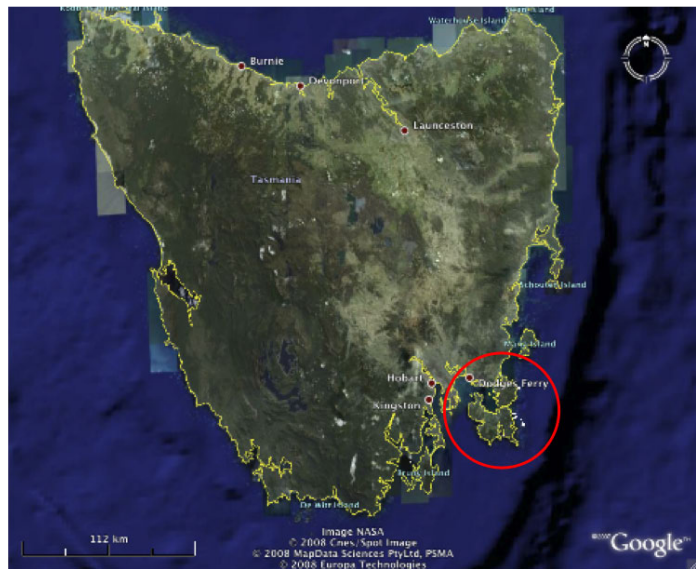

(a) Tasman Peninsula

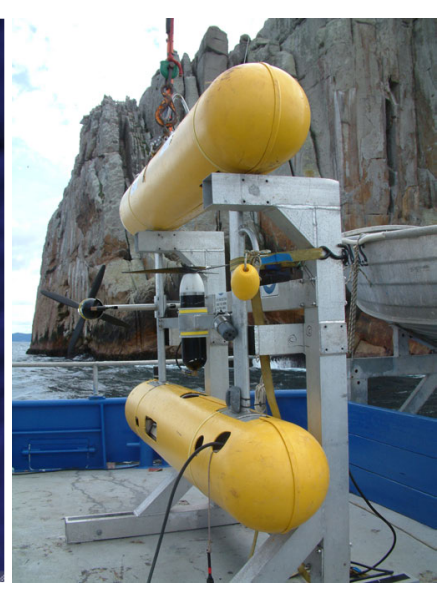

(b) AUV Sirius

Fig. 1 (a) The site of survey work on the Tasman Peninsula in the South East of Tasmania. (b) The vehicle on-board the R/V Challenger prior to deployment. The dolerite cliffs of the peninsula can be seen in the background.

(DEMs) of the study areas. The DEMs were used to determine suitable AUV survey locations and to identify any hazards to operation. At each location, multiple reefs were surveyed at a range of depths from approximately $50 \mathrm{~m}$ to $100 \mathrm{~m}$ depth. Where distinct ectones (e.g. reef to sand) are present, transects were designed to cross transition zones and help determine the uniqueness of ectonal assemblages. Replication depended upon site logistics, however, dive profiles were designed to provide sufficient replication to quantitatively determine abundances of key species/features within depth strata, within reefs, between reefs ( $\mathrm{km}$ to $100 \mathrm{~km}$ scale), and between differing levels of reef complexity.

\section{Biodiversity Hub}

While inshore reef systems are relatively easy to access and describe using methods such as dive surveys, offshore systems have remained relatively unknown because of the expense and complexity of available survey methods. A recent and very significant development contributing to our understanding of the physical environment of shelf habitats has been multibeam sonar and the interpretation of its associated backscatter. This has opened up opportunities for developing predictive capacity in this field where matching biological datasets are available. However, the effectiveness of this technique has yet to be fully tested as an appropriate surrogate for predicting patterns of biodiversity because of the lack of matching biological datasets collected at the same spatial scales and locations as fine scale acous- 
tic surveys. In this context, the AUV deployments reported on here were part of a multi-disciplinary experimental program in eastern Tasmania, where the analysis of covariance is to be undertaken on co-located fine-resolution seabed habitat data, provided by the EM3002 multibeam sonar data, and biological datasets collected at similar spatial scales by Remotely Operated Vehicles (ROVs), Baited Underwater Video systems (BRUVs), towed video and the AUV. This research is being undertaken by the University of Tasmania and Geoscience Australia as part of the Marine Biodiversity Research Hub, which is a collaborative program funded under the Commonwealth Government's Commonwealth Environmental Research Facilities (CERF) Program. Within this Hub, several interrelated projects are designed to develop and test appropriate surrogates for biodiversity and incorporate these into an advanced predictive framework that covers a range of spatial scales [2].

The AUV data enables a finer-scale coupling of biological datasets with multibeam bathymetry than data collected through the use of ROVs, BRUVs and towed video alone, because of geo-referencing errors associated with USBL tracking systems. This additional data is expected to allow scale matching errors to be examined in more detail and allow surrogacy to be examined at the finest possible scale. Ultimately, CERF researchers expect to be able to compare the relative efficiency of using AUV, ROV and towed video systems for shelf habitat biological surveys. In addition, the high resolution images produced by the AUV are expected to significantly enhance the ability to identify taxa, adding finer taxonomic resolution, and hence value, to the data collection.

\section{AUV-based Benthic Habitat Mapping}

One of the key features of the present cruise was the availability of a high resolution optical imaging AUV. The high spatial resolution and capacity to geo-reference the resulting imagery provides an invaluable mechanism for observing the extent and composition of particular benthic habitats. In this case, these data allow for post cruise analysis to validate habitat classification based on backscatter and slope data extracted from the ship-borne multibeam bathymetry.

AUVs are becoming significant contributors to modern oceanography, increasingly playing a role as a complement to traditional survey methods. Large, fast survey AUVs can provide high resolution acoustic multibeam and sub-bottom data by operating a few tens of meters off the bottom, even in deep water $[6,10]$. High resolution optical imaging requires the ability to operate very close to potentially rugged terrain. The Autonomous Benthic Explorer (ABE) has helped increase our understanding of spreading ridges, hydrothermal vents and plume dynamics [16] both using acoustics and vision. The AUV SeaBED [14] is primarily an optical imaging AUV, used in a diverse range of oceanographic cruises including coral reef characterization [13] and surveys of ground fish populations [1]. Recently, the re-

lated AUVs Puma and Jaguar searched for hydrothermal vents under the artic ice [7]. Other AUV systems have been used to explore biophysical coupling, including 
mapping harmful algal blooms [11] and characterising up-welling around canyons [12].

The University of Sydney's Australian Centre for Field Robotics operates an ocean-going AUV called Sirius capable of undertaking high resolution, geo-referenced survey work [15]. This platform is a modified version of the WHOI SeaBED vehicle. This class of AUV has been designed specifically for relatively low speed, high resolution imaging and is passively stable in pitch and roll. The submersible is equipped with a full suite of oceanographic sensors including a high resolution stereo camera pair and strobes, multibeam sonar, a depth sensor, Doppler Velocity Log (DVL) including a compass with integrated roll and pitch sensors, Ultra Short Baseline Acoustic Positioning System (USBL), forward-looking obstacle avoidance sonar, a conductivity/temperature sensor and combination fluorometer/scattering sensor to measure chlorophyll-a, turbidity and dissolved organic matter. The on-board computer logs sensor information and runs the vehicle's low-level control algorithms. Sirius is part of the Integrated Marine Observing System (IMOS) AUV Facility, with funding available on a competitive basis to support its deployment as part of marine studies in Australia.

Navigation underwater is challenging because electromagnetic signals attenuate strongly with distance. Absolute position estimates such as those provided by GPS are therefore not readily available. Simultaneous Localisation and Mapping (SLAM) is the process of concurrently building a feature based map of the environment and using this map to obtain estimates of the location of the vehicle. The SLAM algorithm has seen a considerable amount of interest from the mobile robotics community as a tool to enable fully autonomous navigation [3, 4]. Our current work has concentrated on efficient, stereo based Simultaneous Localisation and Mapping and dense scene reconstruction suitable for creating detailed maps of seafloor survey sites $[8,9]$. These novel approaches, based on Visual Augmented Navigation (VAN) techniques [5], enable the complexity of recovering the state estimate and covariance matrix in a VAN framework to be managed. This has allowed these algorithms to run on significantly larger mapping problems than was previously feasible. These techniques have been used to renavigate the estimated vehicle trajectories using the data collected for this paper.

A typical dive will yield several thousand geo-referenced overlapping stereo pairs. While useful in themselves, single images make it difficult to appreciate spatial features and patterns at larger scales. It is possible to combine the SLAM trajectory estimates with the stereo image pairs to generate 3D meshes and place them in a common reference frame [15]. The resulting composite mesh allows a user to quickly and easily interact with the data while choosing the scale and viewpoint suitable for the investigation. Spatial relationships within the data are preserved and scientists can move from a high level view of the environment down to very detailed investigation of individual images and features of interest within them. This is a useful tool for the end user to develop an intuition of the scales and distributions of spatial patterns, even before any automated interpretation is attempted. Examples of the output of the $3 \mathrm{D}$ reconstructions for dives undertaken on this cruise are included below. 


\section{Deployments}

The deployments undertaken over the course of the 10 day cruise in October 2008 were on shelf reef habitats at depths of between 50 and $100 \mathrm{~m}$ in eastern Tasmanian waters and in estuarine waters in 30 to $50 \mathrm{~m}$ deep in the Huon MPA and around Port Arthur, Tasmania. The vehicle was deployed on 19 dives over the 10 days of operation. During the course of these dives, the vehicle covered in excess of 70 linear kilometers of survey and collected nearly 160,000 high resolution stereo image pairs. Each dive ranged between $2.5 \mathrm{~km}$ and $6.5 \mathrm{~km}$ in total length with an average of $3.7 \mathrm{~km}$ covered per dive travelling at a speed of $0.5 \mathrm{~m} / \mathrm{s}$ or approximately $1 \mathrm{knot}$. Table 1 shows summary statistics for the dives undertaken during the course of this cruise. Dives 1 through 4 were calibration runs undertaken prior to the scientific missions and are not show here.

Figure 2 shows the AUV dive profiles overlaid on the previously collected shipborne mutlibeam bathymetry, focusing on the Tasman Peninsula deployments. The AUV dive profiles were targeting particular rocky reef structures identified in the bathymetry derived from ship-borne multibeam surveys undertaken prior to the cruise. Figure 3 (a) shows two AUV dive profiles over the bathymetry at OHara reef (seen in the middle of Figure 2 (b)) with high resolution multibeam data collected by the vehicle from an altitude of $20 \mathrm{~m}$ over the eastern edge of the reef embedded in the figure. Figure 3 (b) shows details of one of the three dimensional seafloor

Table 1 AUV Tasmania Dive Summary

\begin{tabular}{|c|c|c|c|c|c|}
\hline Dive & Long & $\begin{array}{r}\text { Max Depth } \\
{[\mathrm{m}]}\end{array}$ & $\begin{array}{r}\text { Distance } \\
{[\mathrm{m}]}\end{array}$ & $\begin{array}{l}\text { Avg. Alt. } \\
{[\mathrm{m}]}\end{array}$ & No. Stereo pairs \\
\hline & & & & & \\
\hline-43.0615 & 147.9648 & 67.0 & 5625 & 2.00 & 12262 \\
\hline-43.0631 & 147.9825 & 66.3 & 2947 & 2.00 & 7256 \\
\hline $7-43.084558$ & 147.974086 & 77.9 & 4774 & 2.00 & 11278 \\
\hline $8-43.094119$ & 148.024267 & 85.9 & 2817 & 2.10 & 7262 \\
\hline $9-43.120019$ & 148.05373 & 90.9 & 2652 & 2.08 & 6336 \\
\hline $10-43.119798$ & 148.047008 & 88.5 & 2717 & 2.06 & 6406 \\
\hline $11-43.119126$ & 148.038401 & 83.3 & 2660 & 1.97 & 6727 \\
\hline $12-43.120581$ & 148.03754 & 84.0 & 2875 & 2.00 & 6787 \\
\hline $13-43.123972$ & 148.053974 & 96.3 & 2759 & 2.16 & 6870 \\
\hline $14-43.119887$ & 148.045257 & 89.5 & 2995 & 2.06 & 7737 \\
\hline $15-43.040462$ & 147.955845 & 57.8 & 4559 & 1.99 & 10563 \\
\hline $16-42.956996$ & 148.005154 & 76.4 & 6542 & 2.05 & 15521 \\
\hline $17-43.165023$ & 147.874164 & 52.7 & 4290 & 1.99 & 9768 \\
\hline $18-43.18674$ & 147.88763 & 32.4 & 3889 & 2.02 & 8863 \\
\hline $19-42.913151$ & 148.003898 & 60.0 & 6300 & 2.00 & 15162 \\
\hline $20-43.084558$ & 147.974086 & 76.9 & 2780 & 2.04 & 6564 \\
\hline $21-43.29307$ & 147.129389 & 45.9 & 4619 & 2.00 & 9655 \\
\hline $22-43.327701$ & 147.166552 & 35.8 & 1153 & 1.51 & 2548 \\
\hline $23-43.270816$ & 147.124965 & 33.0 & 3200 & 1.50 & 1623 \\
\hline TOTAL & & & 70153 & & 159188 \\
\hline
\end{tabular}




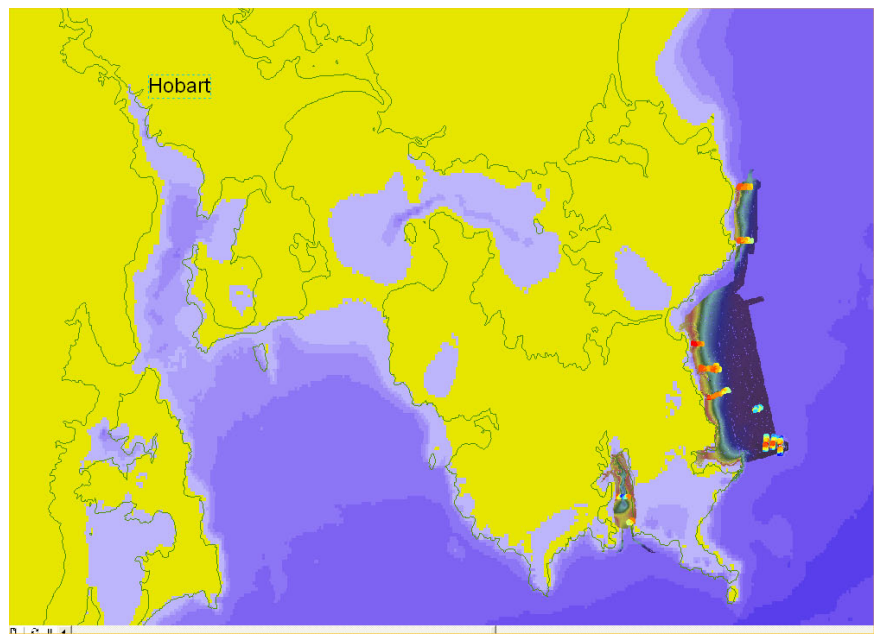

(a) Tasman peninsula deployments

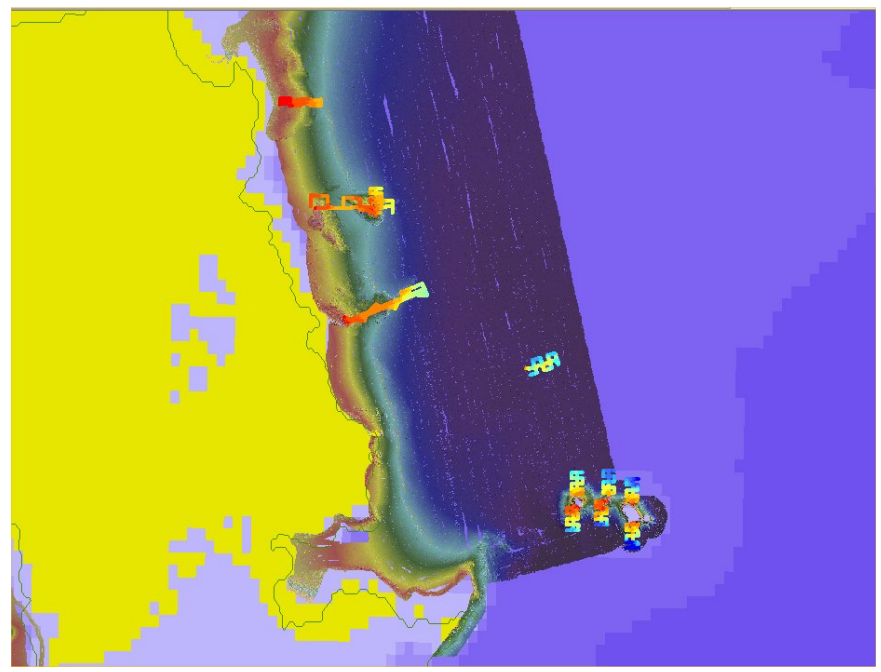

(b) Fortescue Bay

Fig. 2 Tasmanian Deployments 2008 illustrating the prior multibeam bathymetry available with AUV dive profiles overlaid (ship-borne multibeam sonar image courtesy of Geoscience Australia). The AUV profiles are colour coded by depth.

reconstructions generated using the combined SLAM and stereo meshes for one of these dives.

Figure 4 shows dive profiles for six dives undertaken around the Hippolytes, a rocky island located group approximately $4 \mathrm{~km}$ offshore. These areas, in approximately $90 \mathrm{~m}$ of water, were expected to feature high levels of biodiversity. Finally, Figure 5 shows high resolution multibeam bathymetry collected by the vehicle in 
the Huon MPA showing a series of pockmarks mapped using the vehicle's on-board multibeam sonar at an altitude of $20 \mathrm{~m}$ during dive 23 . These pockmarks had been identified in prior ship borne multibeam surveying but were targeted by the AUV to generate higher resolution bathymetry as well as undertaking a number of visual passes at an imaging altitude of $2 \mathrm{~m}$. The visual imagery revealed that these pockmarks, surrounded by muddy substrate, were filled with algal growth. Further investigation using divers is planned to determine the nature of this growth.

The data gathered during the AUV dives will form a baseline at or near the time of protection for newly designated MPAs. This will allow changes related to protection, time and climate change to be assessed through time. This dataset will underpin proposals for ongoing research on the respective MPAs (Commonwealth and State) with respect to both ecosystem effects of fishing and climate change in an otherwise poorly understood ecostystem. The AUV, ROV and BRUV surveys will also provide, for the first time, a detailed quantitative inventory of the benthic faunal assemblages associated with temperate shelf reef systems in this region, and of the MPAs themselves. Shelf reef assemblages of this region remain relatively undescribed with respect to the quantitative composition of dominant species or taxonomic groupings, therefore providing that initial baseline description represents a significant contribution of this study. Like inshore systems, shelf reef assemblages in this region are influenced by fishing (e.g. scalefish and lobster), introduced species and climate change, yet nothing is currently known of the interactions occurring in these systems.

\section{Conclusions and Future Work}

This paper has reported on an expedition taken to survey biological assemblages associated with deep water, rocky reef systems off the coast of the Tasman peninsula and in the Huon MPA. Although the detailed analysis of the data and comparison against the benthic habitat classification based on sonar backscatter and slope is ongoing, we have illustrated how the data collected by the AUV is complementary to ship-borne multibeam.

We are currently preparing to return to Tasmania in early 2009 to undertake additional deployments associated with further multibeam swath mapping and to target urchin barrens and scallop fisheries. The impact of fishing and climate change on these poorly understood habitats stands to derive significant benefit from the detailed, optical surveying capabilities of tools such as the AUV.

\section{Acknowledgment}

This work is supported by the ARC Centre of Excellence programme, funded by the Australian Research Council (ARC) and the New South Wales State Government, and the Integrated Marine Observing System (IMOS) through the DIISR National Collaborative Research Infrastructure Scheme. The authors would like to thank the 


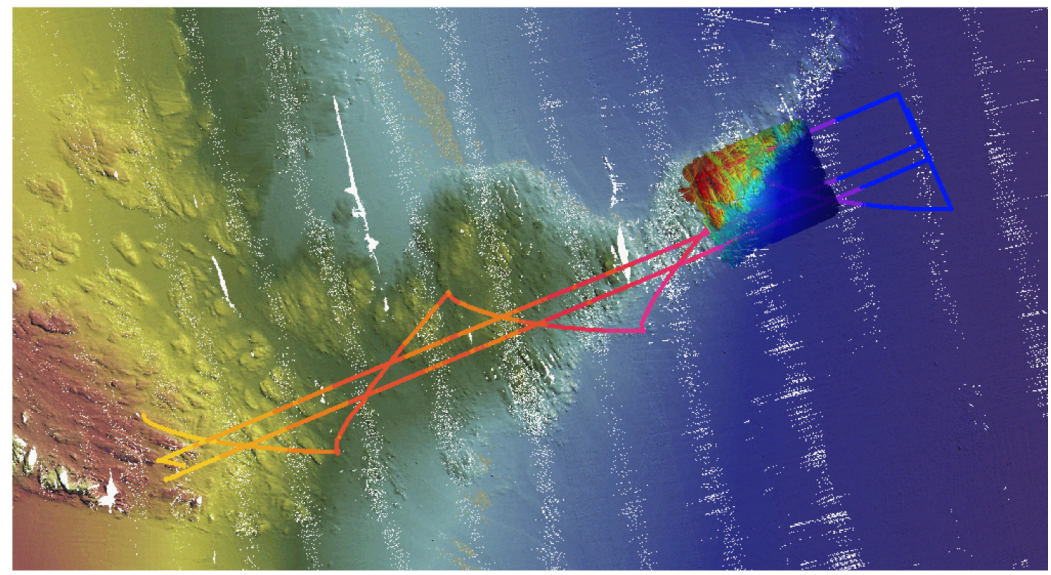

(a) Ohara reef bathymetry

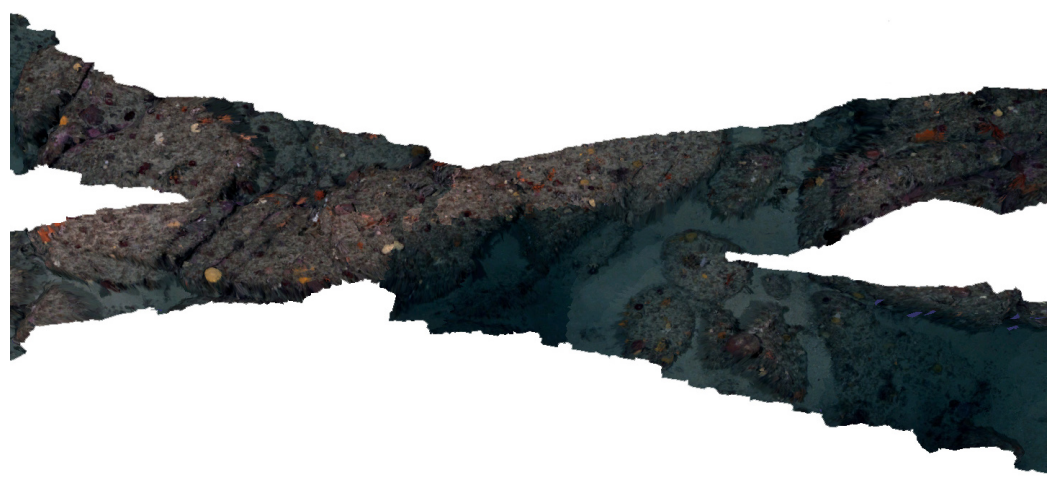

(b) $3 \mathrm{D}$ reconstruction

Fig. 3 (a) Ohara reef bathymetry with two AUV imaging dive profiles (Dive 7 and 20) overlaid (ship-borne multibeam sonar image courtesy of Geoscience Australia). The long axis of these dives are $2 \mathrm{~km}$ in length. The AUV dive profiles are colour coded by measured depth. The colour coding between the AUV depth and the bathymetry are not consistent in order for the dive profile to stand out. Detailed bathymetry collected by the vehicle from a $20 \mathrm{~m}$ altitude at the eastern edge of the reef is overlaid on the orginal ship-borne bathymetry showing the interface between the rocky reef and the deeper, sandy substrate. The AUV bathymetric data is gridded at $0.5 \mathrm{~m}$ resolution. (b) Details of one of the SLAM loop closure points identified during Dive 7 at OHara reef. The vehicle path generated using SLAM has been used to place the stereo meshes into space and the resulting mesh has been texture mapped using the images. As can be seen, the loop closure has been successful, resolving the detailed structure of the scene in spite of the vehicle having travelled nearly $2 \mathrm{~km}$ between the two passes over this area.

Captain and crew of the R/V Challenger. Their sustained efforts were instrumental in facilitating successful deployment and recovery of the AUV. Thanks to Justin Hulls and Jan Seiler for help and support on-board the ship. Thanks also to Dr. Vanessa Lucier of TAFI for providing the ship borne multibeam and to Dr. Tara Anderson 


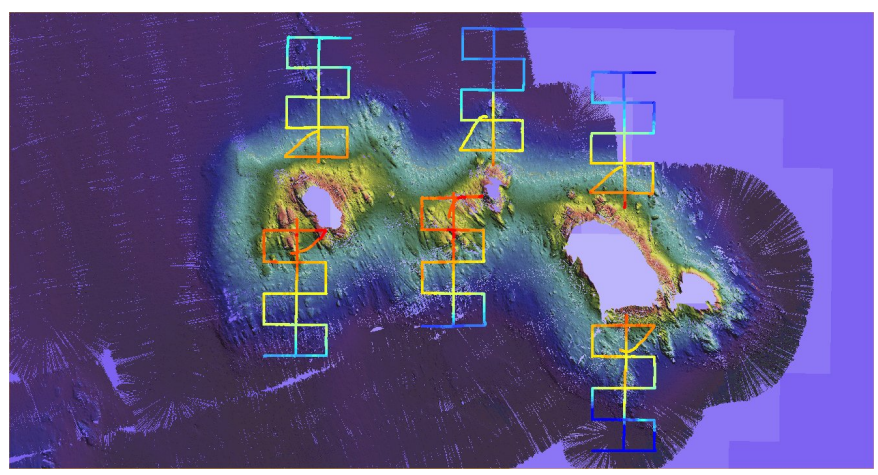

Fig. 4 Dive profiles for dives 8 through 13 around the Hippolytes, a series of islands located some $4 \mathrm{~km}$ offshore of the Tasman peninsula (ship-borne multibeam sonar image courtesy of Geoscience Australia).

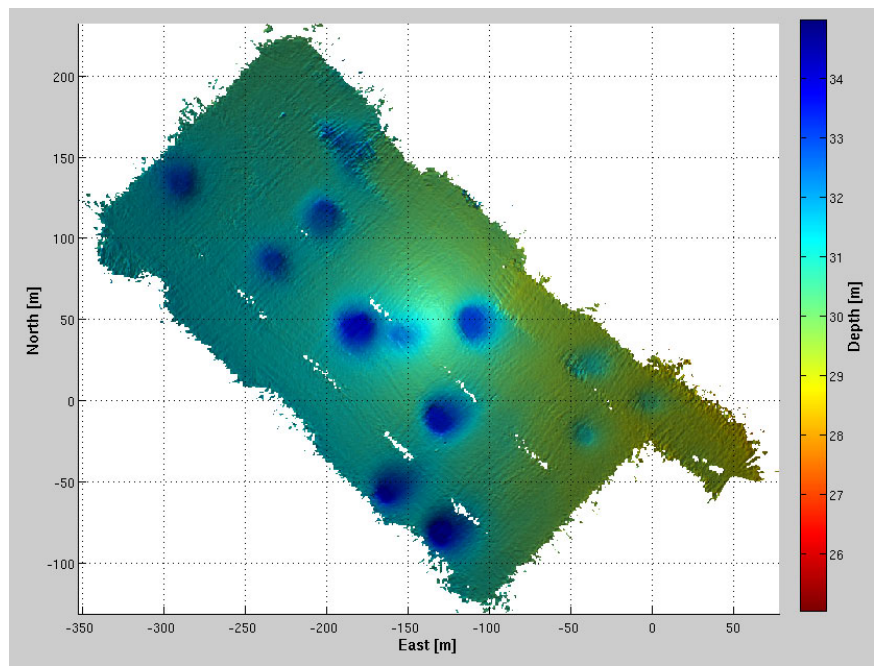

Fig. 5 A series of pockmarks mapped using the vehicle's on-board multibeam sonar at an altitude of $20 \mathrm{~m}$ during dive 23 in the Huon MPA.

from Geoscience Australia for her contribution to the AUV transect design for the surveys. The ship-borne multibeam sonar data were collected, processed and gridded to produce DEMs by Geoscience Australia. We also acknowledge the help of all those who have contributed to the development and operation of the AUV, including Duncan Mercer, George Powell, Ian Mahon, Matthew Johnson-Roberson, Stephen Barkby, Ritesh Lal, Paul Rigby, Jeremy Randle, Bruce Crundwell and the late Alan Trinder. 


\section{References}

[1] Clarke M, Singh H, CGoldfinger, Andrews K, Fleischer G, Hufnagle L, Pierce S, Roman C, Romsos C, Tolimieri N, WWakefield, York K (2006) Integrated mapping of West Coast groundfish and their habitat using the Seabed AUV and the ROPOS ROV. EOS Trans AGU 87(36):Ocean Sci. Meet. Suppl., Abstract OS46G-12

[2] Commonwealth Environmental Research Facilities (CERF) Marine Biodiversity Hub (2009) URL http://www.marinehub.org/index.php/site/home

[3] Dissanayake M, Newman P, Clark S, Durrant-Whyte H, Csobra M (2001) A solution to the simultaneous localization and map building (SLAM) problem. In: IEEE Transactions on Robotics and Automation, vol 17(3), pp 229-241

[4] Durrant-whyte H, Bailey T (2006) Simultaneous localisation and mapping (SLAM): Part I the essential algorithms. Robotics and Automation Magazine 13:99-110

[5] Eustice R, Singh H, Leonard J, Walter M (2006) Visually mapping the RMS Titanic: conservative covariance estimates for SLAM information filters. Intl J Robotics Research 25(12):1223-1242

[6] Grasmueck M, Eberli GP, Viggiano DA, Correa T, Rathwell G, Luo J (2006) Autonomous underwater vehicle (AUV) mapping reveals coral mound distribution, morphology, and oceanography in deep water of the straits of Florida. Geophysical Research Letters 33:L23,616

[7] Kunz C, Murphy C, Camilli R, Singh H, Eustice R, Roman C, Jakuba M, Willis C, Sato T, Nakamura K, Sohn R, Bailey J (2008) Deep sea underwater robotic exploration in the ice-covered arctic ocean with AUVs. In: IEEE/RSJ Intl. Workshop on Intelligent Robots and Systems, pp 3654-3660

[8] Mahon I (2008) Vision-based navigation for autonomous underwater vehicles. $\mathrm{PhD}$ thesis, University of Sydney

[9] Mahon I, Williams SB, Pizarro O, Johnson-Roberson M (2008) Efficient view-based SLAM using visual loop closures. IEEE Transactions on Robotics and Automation 24(5):1002-1014

[10] Marthiniussen R, Vestgard K, Klepaker R, Storkersen N (2004) HUGIN-AUV concept and operational experiences to date. In: OCEANS '04. MTTS/IEEE TECHNO-OCEAN '04, vol 2, pp 846-850 Vol.2

[11] Robbins I, Kirkpatrick G, Blackwell S, Hillier J, Knight C, Moline M (2006) Improved monitoring of HABs using autonomous underwater vehicles (AUV). Harmful Algae 5(6):749-761

[12] Ryan J, Chavez F, Bellingham J (2005) Physicalbiological coupling in monterey bay, california: topographic influences on phytoplankton ecology. Marine Ecology Progress Series 287:23-32

[13] Singh H, Armstrong R, Gilbes F, Eustice R, Roman C, Pizarro O, Torres J (2004) Imaging Coral I: Imaging Coral Habitats with the SeaBED AUV. Subsurface Sensing Technologies and Applications 5:25-42

[14] Singh H, Can A, Eustice R, Lerner S, McPhee N, Pizarro O, Roman C (2004) SeaBED AUV offers new platform for high-resolution imaging. EOS, Transactions of the AGU 85(31):289,294-295

[15] Williams S, Pizarro O, Mahon I, Johnson-Roberson M (2008) Simultaneous localisation and mapping and dense stereoscopic seafloor reconstruction using an AUV. In: Proc. of the Int'l Symposium on Experimental Robotics

[16] Yoerger D, Jakuba M, Bradley A, Bingham B (2007) Techniques for deep sea near bottom survey using an autonomous underwater vehicle. The International Journal of Robotics Research 26:41-54 